\title{
Rupture of uterine vessel by twisted leiomyoma causing massive intraperitoneal haemorrhage and hypovolemic shock
}

Pathiraja R P1

\begin{abstract}
A 26 year old nullipara who was 1 diagnosed with multiple uterine leiomyomata, presented in the emergency care unit with acute lower abdominal pain and slight vaginal bleeding. On admission to hospital, three hours after the onset of pain, she was very pale her skin was cold and was feeling faint, the temperature was $37.5^{\circ} \mathrm{C}$ pulse 92 beats per minute, blood-pressure 95/60 $\mathrm{mmHg}$ and respiration was 36 per minute. The chest and heart were normal. The abdomen was distended with generalized tenderness and rigidity, most pronounced over the suprapubic region. Pregnancy test was negative. Ultrasound scan revealed multiple uterine leiomyomata and free fluid in the peritoneal cavity. The diagnosis of acute abdomen due to haemoperitoneum was made.
\end{abstract}

The patient underwent an explorative laparotomy. The peritoneal cavity was full of blood. The tubes and ovaries were normal. Uterus was enlarged with multiple leiomyomata in varying sizes (Figure1).

A fibroid $5 \times 5 \times 3 \mathrm{~cm}$ on the posterior surface of the uterus was partly detached from its bed, leaving a small cavity as if it had been partially enucleated. The torn area was bleeding freely. Myomectomy was done and the raw area was repaired (Figure 2 and 3 ).

${ }^{1}$ Senior Lecturer, Faculty of Medical Sciences, University of Sri Jayewardenepura,

Nugegoda, Sri Lanka.

Correspondence: R P Pathiraja

E-mail: ramya_pathiraja@yahoo.com

Competing interests: None

\section{Figure 1. Uterus with multiple fibroids $4 \times 5.3$ inches}

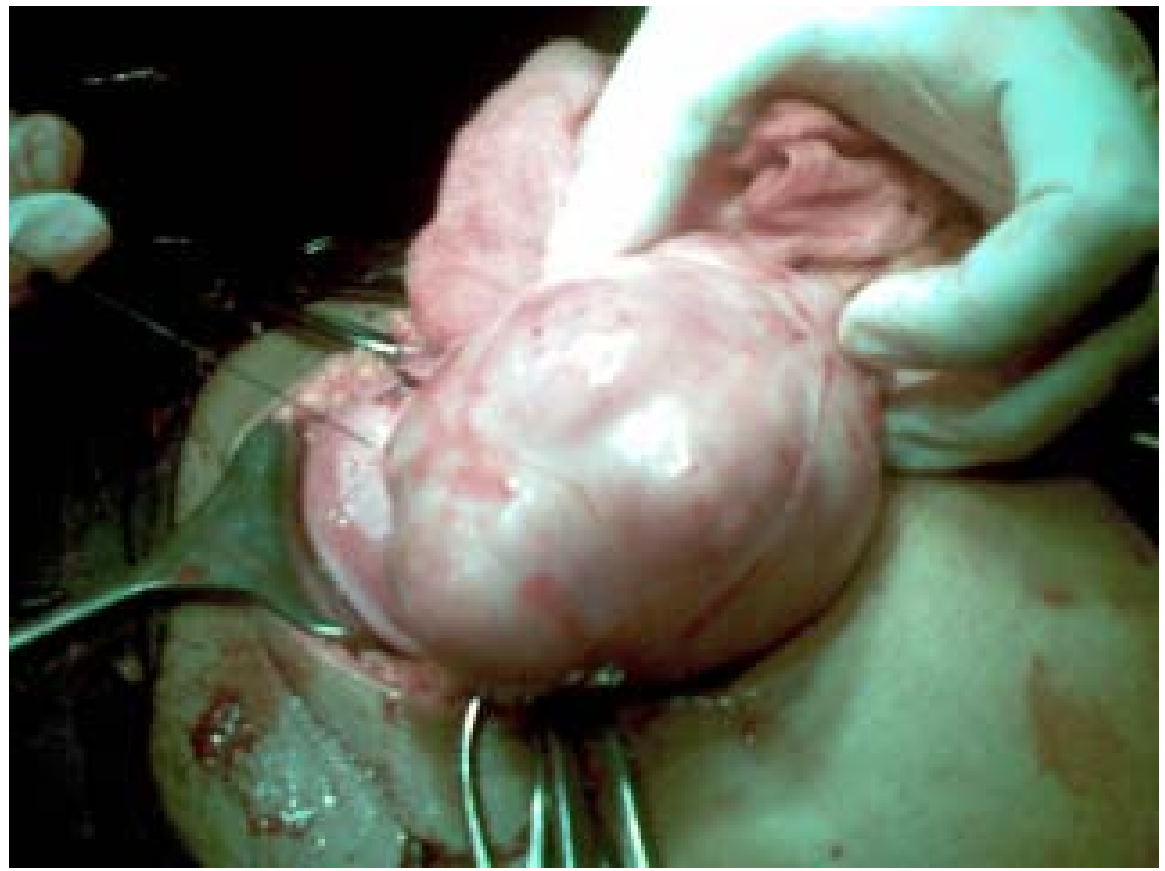

Figure 2. Posterior surface of the uterus where the fibroid was detached $4 \times 5.3$ inches

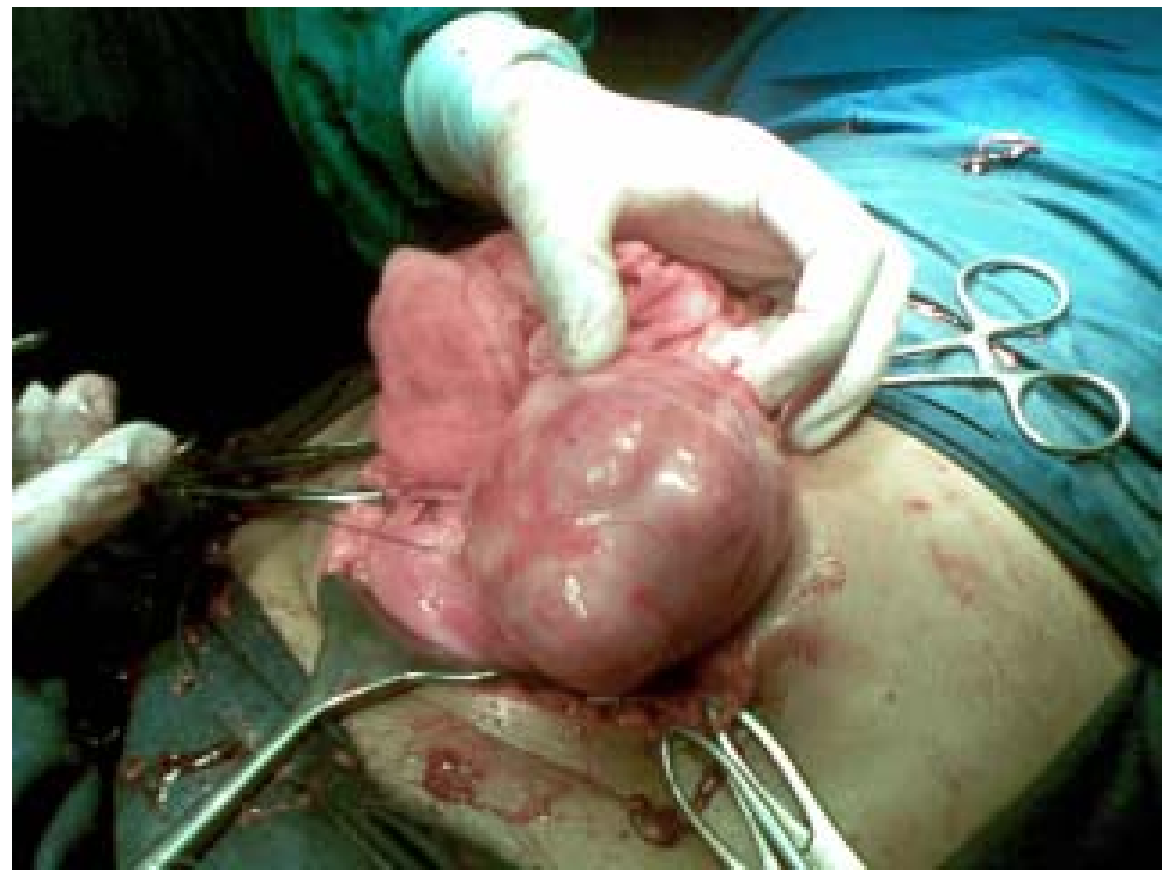




\section{Figure 3. Following the repair of the posterior surface of the uterus where the fibroid was detached $4 \times 5.3$ inches}

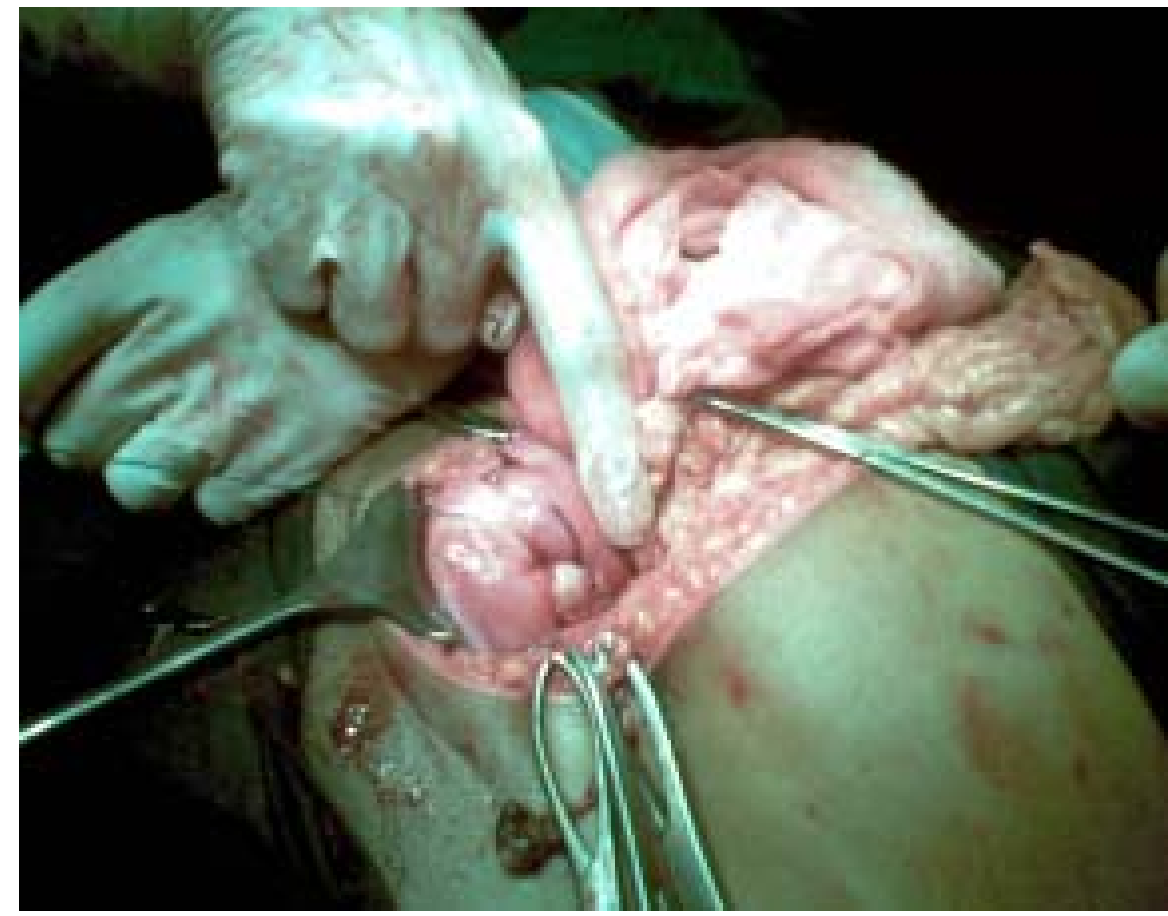

Figure 4. After removal of all the fibroids $4 \times 5.3$ inches

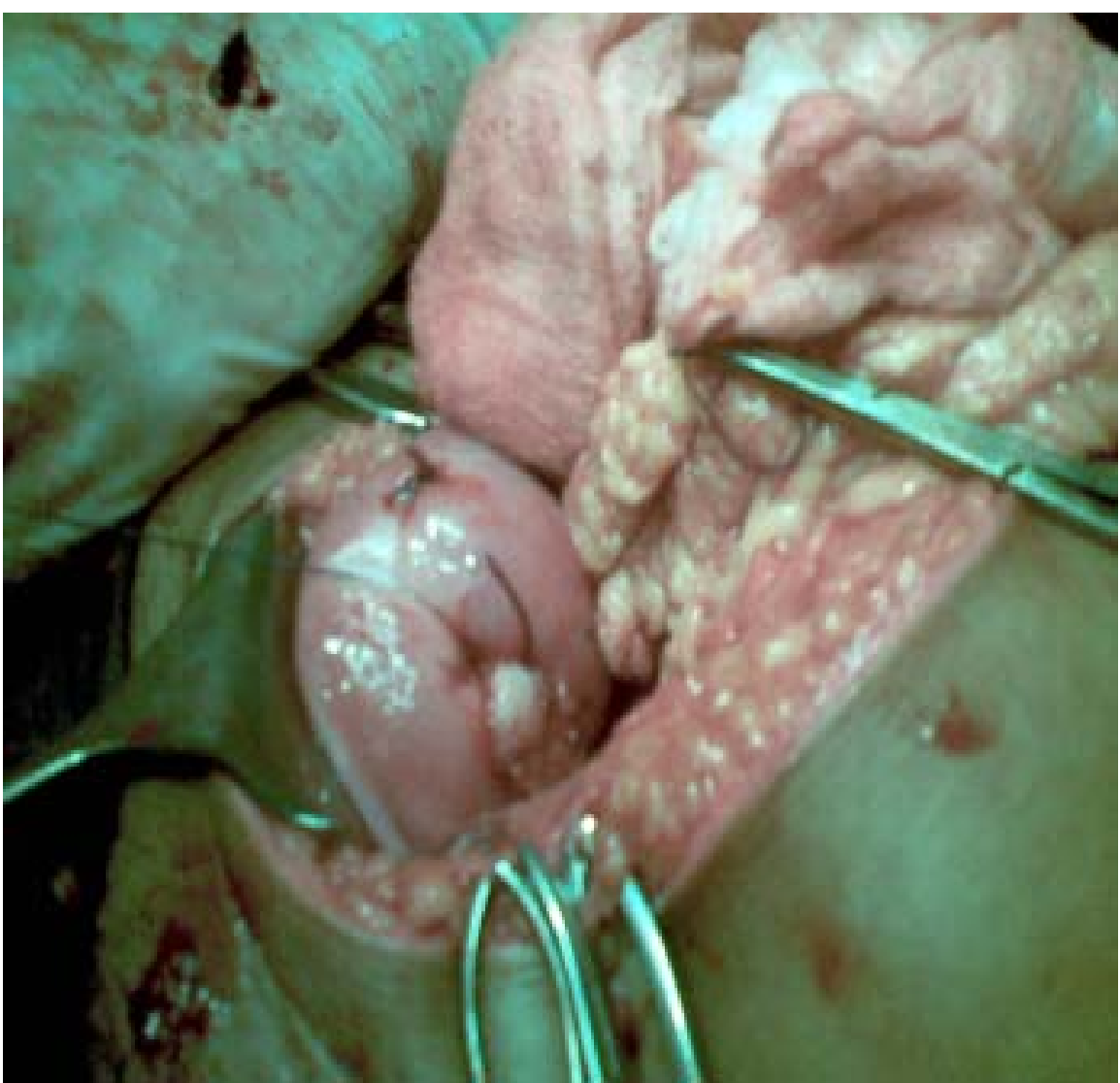

Other fibroids were also removed (Figure 4). The patient's postoperative course was uneventful. The histology reported as leiomyomata containing haemorrhages with areas of oedema and thrombosis. There were no abnormal cellular mitosis.

\section{Discussion}

Haemoperitoneum associated with uterine leiomyoma is very rare. In most cases the bleeding occurs as a result of rupture of a superficial vein ${ }^{1}$ and occasionally due to avulsion by trauma ${ }^{2}$ or due to rupture of fibroid ${ }^{3}$. Rupture of a vein on the surface of a uterine fibromyoma was first reported by Von Rokitansky $\mathrm{C}^{4}$ the condition was discovered at necropsy of a girl who had died from intra abdominal haemorrhage.

Haemoperitoneum from subserous fibroid due to spontaneous avulsion is reported in only three cases ${ }^{5}$, the first case was reported by Shelly in 1931 and Cottalorda in 1935 reported another case in whom gangrenous appendix was found, adherent to the avulsed fibroid at laporatomy carried out for suspected appendicitis ${ }^{5,6}$ and the third case was reported by Pappa Dasari in $2005^{6}$.

There has been much speculation regarding the factors which precipitate rupture of the veins on the surface of fibroid tumours. Twisting of the pedicle of a subserous myoma, a direct blow to the abdomen, abdominal massage, a fall, violent coitus, injury to a vein by the sacral promontory and an increase in intraabdominal pressure, which may then increase the venous pressure have all been blamed. Increased venous congestion and rupture of the tumour during menstruation and pregnancy has been reported by Mattison and $\mathrm{Yeh}^{7}$.

Trauma associated with pelvic fractures can also cause avulsion of the pedunculated leiomyoma ${ }^{3,5}$. Posterior location of a bleeding vein supports the theory of direct-contact injury from the promontory of the sacrum. Degeneration of a leiomyoma 
during pregnancy and puerperium cause spontaneous perforation and bleeding into the abdomen ${ }^{8,9}$.

Like our patient, twisting of the pedicle of a subserous myoma was held responsible in 10 out of 60 cases in a study of world literature by Hasskari ${ }^{10}$. This condition produces acute abdominal symptoms which make an exploratory laparotomy imperative. The usual diagnosis in these cases were ectopic gestation, rupture or torsion of an ovarian cyst or fibroid tumour, ruptured viscus, or acute appendicitis, acute pancreatitis or ruptured spleen. Very seldom has the diagnosis been made preoperatively, for example, in only 4 of the 60 cases collected by Hasskari ${ }^{10}$.

\section{Treatment}

The treatment consists of immediate explorative laparotomy ${ }^{11}$ and myomectomy in most cases, as these patients are gravely ill and also comparatively young ${ }^{12,13}$. Subtotal hysterectomy has been carried out in some cases, because of the poor general condition of the patient ${ }^{11}$. The woman's age must be considered in decisions regarding hysterectomy ${ }^{9}$. However, when the condition is critical or when there are multiple uterine leiomyomas and many superficial blood vessels, hysterec- tomy may be the only appropriate option $^{14,15}$.

In one case, owing to the desperate state of the patient at the time of the operation, ligation of the bleeding vein was all that was done ${ }^{10}$.

We report this case due to rarity and life threatening nature of the condition. As surgical management is needed in an emergency, surgeons should be aware of this rare but severe complication in patients with known leiomyomas.

In conclusion, definitive, preoperative diagnosis of uterine vessel rupture by twisting fibroids is difficult; explorative laporatomy is both diagnostic and therapeutic in this rare, life threatening condition.

\section{REFERENCES}

1. Buttery BW. Spontaneous haemoperitoneum complicating fibromyoma. Aust N Z J Obstet Gynaecol 1972; 12: 210-3.

2. Sule AZ. Traumatic rupture of uterine fibroid: an uncommon cause of post-traumatic haemoperitoneum. West Afr J Med 2000; 19; 158-9.

3. Drutman J, Fruechte DM. Haemoperotoneum due to traumatic avulsion of a pedunculated uterine leiomyoma. $\mathrm{Br}$ Med $\mathrm{J}$ 1967; 3: 723-4.

4. Von Rokitansky C, Lehrbuch der pathologischen Anatomie. wien: Bramuller. w. 1861.
5. Venables CW craft IL. Haemoperitoneum after traumatic avulsion of uterine fibroid. Br Med J 1967; 16: 723-4.

6. Papa D, Maurya DK, Haemoperitoneum associated with fibroid uterus. The $\mathrm{J}$ of Obstetrics and Gynaecology of India November/December 2005; 55(6): 553-4.

7. Mattison DR, Yeh SY, Haemoperitoneum from rupture of uterine vein overlying a leiomyoma. Am J Obstet 1980; 136: 415-16.

8. Saidi F, Constable JD, Ulfelder H. Massive intraperitoneal haemorrhage due to uterine fibroids. Am J Obstet Gynaecol 1961; 82: 367-74.

9. Grisaru D, Bar-Am, Lessing JB. Spontaneous perforation of a degenerative nongestational uterine leiomyoma. Obstet Gynaecol1996; 87(5 pt 2): 882.

10. Hasakari WF, In: proc. Staff meet. Mayo clinic; 1949; P. 207.

11. Varras M, Antoniou S, Samarach, Frakala S, Angelidou-Manika Z, Paissios P. Intraperitoneal haemorrhage secondary to perforation of uterine fibroid after cystic generation. Unusual CT findings resembling malignant pelvic tumour: case report. Eur $\mathrm{J}$ Gynaecol Oncol 2002; 23(6): 565-8.

12. Badaway AH. Brit Med J, 1961; 1: 717.

13. Bibgby MAM, Brit Med J. 1960; 2: 580.

14. Deopuria RH. Haemoperitoneum secondary to rupture of coronary veins on a fibroid uterus (review). J Obstet Gynaecol Indian1970; 20: 409-13.

15. Saidi F, Constable JD, Ulfelder H. Masssive intraperitoneal haemorrhage due to uterine fibroids. Am J Obstet Gynaecol 1961; 82: 367-74. 